\title{
Efecto de la Fibrina Rica en Plaquetas en la Curación de los Tejidos Blandos de Alveolos Post Exodoncia Atraumática. Un Ensayo Clínico Controlado Aleatorizado Cruzado a Ciego Simple
}

\author{
Effect of the Platelet-Rich Fibrin on the Healing of the Soft Tissues of Sockets \\ after Atraumatic Exodontics. A Single-Blind Cross-Randomized Controlled Clinical Trial
}

Miguel Travezán-Moreyra'; AugustoAguirre-Aguilar ${ }^{1}$ \& Heber Arbildo-Vega a $2,3,4$

TRAVEZÁN-MOREYRA, M.; AGUIRRE-AGUILAR, A. \& ARBILDO-VEGA, H. Efecto de la fibrina rica en plaquetas en la curación de los tejidos blandos de alveolos post exodoncia atraumática. Un ensayo clínico controlado aleatorizado cruzado a ciego simple. Int. J. Odontostomat., 15(1):240-247, 2021.

RESUMEN: El objetivo de este estudio fue determinarel efecto de la fibrina rica en plaquetas (FRP) en la curación de los tejidos blandos de alveolos post exodoncia atraumática. El presente es un ensayo clínico controlado aleatorizado a ciego simple y de diseño cruzado. Se llevó a cabo en el Servicio de Odontología del Hospital Distrital Santa Isabel del Porvenir - Perú, durante los años 2016 y 2017. La muestra estuvo conformada por 51 pacientes cuyos alveolos post exodoncia fueron divididos de forma aleatoria en 2 grupos, cada paciente firmó un consentimiento informado. Al grupo A (control) se le dejó con un coágulo de sangre para su curación normal y al grupo B (experimental) se le administró FRP (como tapón y membrana), obtenido según el protocolo de Choukroun. Para determinar el efecto del FRP en la curación de los tejidos blandos de los alveolos post exodoncia atraumática se utilizó el índice de Landry. Las medidas se realizaron a los 7 y 14días después de la cirugía.Para la comparación de cada una de las variables del estudio se utilizó el Test de Mc Nemar y el Test exacto de Fisher. La significación estadística fue del $5 \%$. Al comparar ambos grupos en la curación de los tejidos blandos de alveolos post exodoncia atraumática se encontró que existe una diferencia estadísticamente significativa a los 7 y 14 días después de la cirugía $(p<0.05)$. Al comparar ambos grupos en la curación de los tejidos blandos de alveolos post exodoncia atraumática según grupo etáreo y el sexo se encontró que no existe una diferencia estadísticamente significativa a los 7 y 14días después de la cirugía $(p>0.05)$. La FRP presenta un efecto positivo en la curación de los tejidos blandos de alveolos post exodoncia atraumática de forma independiente del sexo y la edad.

PALABRAS CLAVE: fibrina rica en plaquetas, FRP, alveolo post exodoncia, curación de la herida, tejidos blandos.

\section{INTRODUCCIÓN}

Actualmente en la odontología, uno de los problemas más frecuentes, es la conservación de la cresta alveolar residual después de los procedimientos rutinarios de exodoncia, el cual conlleva a problemas a futuro para la colocación de implantes dentales o su rehabilitación (Srinivas et al., 2018). La exodoncia dental es un procedimiento común para el tratamiento de la caries dental avanzada, fracturas complicadas, enfermedades periodontales, infecciones y creación de espacio ortodóntico (Buchwald \& Kocher, 2013; Gonda \& Mac Entee, 2013; Alzahrani et al., 2017).

\footnotetext{
1 Facultad de Estomatología, Universidad Nacional de Trujillo. Trujillo, Perú.

${ }^{2}$ Facultad de Odontología, Universidad San Martín de Porres. Chiclayo, Perú.

${ }^{3}$ Escuela de Odontología, Universidad Particular de Chiclayo. Chiclayo, Perú.

${ }^{4}$ Centro de Salud Odontológico San Mateo. Trujillo, Perú.
} 
TRAVEZÁN-MOREYRA, M.; AGUIRRE-AGUILAR, A. \& ARBILDO-VEGA, H. Efecto de la fibrina rica en plaquetas en la curación de los tejidos blandos de alveolos post exodoncia atraumática. Un ensayo clínico controlado aleatorizado cruzado a ciego simple. Int. J. Odontostomat., 15(1):240-247, 2021.

La prevención de la pérdida ósea alveolar post exodoncia, fue descrita por primera vez por Greenstein y Ashman y Bruins en el año 1985. La cicatrización o curación de alvéolo post exodoncia comprende la remodelación del hueso y de los tejidos blandos con cambios dimensionales máximos que se producen durante los primeros 3 meses. El plasma rico en plaquetas (PRP) se desarrolló inicialmente en la década de 1970 demostrando que producía un aumento en la tasa de maduración ósea y una mejoraen la densidad ósea, además de promover la cicatrización de heridas y el sellado de tejidos. Sin embargo, las controversias en la literatura con respecto a los beneficios y resultados clínicos del uso de PRP no se pueden ignorar. La fibrina rica en plaquetas (FRP) es una segunda generación de factores de crecimiento autólogos que se prepara a partir de sangre autógena no anticoagulante cuando se centrifuga; se desarrolló por primera vez en Francia como una alternativa terapéutica al PRP para superar muchas de sus limitaciones. La FRP es fácil de preparar, no es tóxica, es biocompatible con los tejidos vivos fomenta la curación y se propone que se asocie con una organización temprana y efectiva de la sustancia ósea y el porcentaje de volumen óseo y es relativamente más económica (Choukroun \& Diss, 2006; Dohan \& Choukroun, 2006; Kutkut \& Andreana, 2012; Marelli \& Tatullo, 2013; Alzahrani et al.; Srinivas et al.).

Los alvéolos post exodoncia pasan por una curación fisiológica que implica un proceso complejo de migración y maduración de células óseas que conduce a una reabsorción y remodelación de la cresta alveolar, el cual produce cambios marcados en la altura y el ancho de la misma, con un promedio de 0,7$1,5 \mathrm{~mm}$ y de 4,0-4,5 $\mathrm{mm}$ de reabsorción ósea vertical y horizontal respectivamente. La mayoría de estas alteraciones dimensionales se producen en los primeros 3 meses posteriores a la exodoncia (Cardaropoli \& Araujo, 2003; Araujo \& Lindhe, 2005; Jiing et al., 2011; Alzahrani et al.; Srinivas et al.). La reabsorción ósea en los alvéolos post exodoncia mandibulares son mayores durante el primer año, aunque se han reportado variaciones de hasta 4 veces másen algunas personas durante un período de 14 meses (Chan et al, 2013; Srinivas et al.).

Se han usado muchos métodos durante las últimas dos décadas para mantener la arquitectura de la cresta alveolar residual, como el uso de hueso, sustitutos óseos y tapones de colágeno empaquetados en los alvéolos post exodoncia sellados con o sin isocianoacrilato; así como también, el empleo de con- centrados de plaquetas autólogas que incluyen al PRP y laFRP (Rutherford \& Niekrash, 1992; Norton \& Wilson, 2002; Forum \& Cho, 2002; Vance \& Greenwell, 2004; Zhang \& Wang, 2007; Chan et al.; Alzahrani et al.; Srinivas et al.). Los concentrados de fibrina autólogos se consideran los mejores para evitar las infecciones cruzadas, sin embargo, su uso sigue siendo muy limitado debido a la falta de competencia del operador y a una comprensión completa de los procesos inherentes. Además, los informes con respecto a la eficacia clínica del uso de concentrados de plaquetas (como la FRP) en la curación de los alvéolos post exodoncia han sido controvertidos (Simonpieri et al., 2009, 2012; Alzahrani et al.; Srinivas et al.). Por todo lo anteriormente mencionado, el propósito del presente estudio fue el de determinar el efecto de la FRP en la curación de los tejidos blandos de alvéolos post exodoncia atraumática.

\section{MATERIAL Y MÉTODO}

Se realizó un estudio analítico, experimental, ensayo clínico controlado aleatorizado y diseño cruzado. La muestra estuvo compuesta por los pacientes que acudieron al Servicio de Odontología del Hospital Distrital Santa Isabel del Porvenir - Perú y que requirieron realizarse exodoncias de durante los años 2016 y 2017. El tipo de muestreo fue probabilístico aleatorio simple.

Los criterios de inclusión para el estudio fueron: pacientes de ambos sexos, que acudieron al Servicio de Odontología del Hospital Distrital Santa Isabel del Porvenir - Perú los años 2016 y 2017, que requerían como mínimo dos dientes indicados para exodoncias, pacientes con un aparente buen estado de salud general y que aceptaran realizarse el tratamiento con el uso del FRP bajo consentimiento informado. Los criterios de exclusión fueron: pacientes con riesgo sistémico no controlado, gestantes o en periodo de lactancia, pacientes con enfermedades sanguíneas, con medicación que afecte la coagulación sanguínea o con bifosfonatos, con alguna incapacidad física o mental, con alguna patología dento-alveolar o periodontal, menores de 18 años, pacientes con signos de displasia o lesiones precancerosas.

Para la realización del estudio se tuvo en cuenta la declaración de Helsinki (2013) de la AMM, de un jurado perteneciente a la Escuela de Post Grado del Área de Ciencias Médicas de la Universidad Nacional 
TRAVEZÁN-MOREYRA, M.; AGUIRRE-AGUILAR, A. \& ARBILDO-VEGA, H. Efecto de la fibrina rica en plaquetas en la curación de los tejidos blandos de alveolos post exodoncia atraumática. Un ensayo clínico controlado aleatorizado cruzado a ciego simple. Int. J. Odontostomat., 15(1):240-247, 2021.

de Trujillo (UNT), de la Ley General de Salud en el Perú (Ley $\left.n^{\circ} 26842\right)$ y de la autorización de cada participante en el estudio (consentimiento informado). Se calculó el tamaño de la muestra y se obtuvo que se requirieron 51 pacientes.

Se realizó, en primer lugar, los trámites documentarios correspondientes para conseguir la autorización del director del Hospital Distrital Santa Isabel del Porvenir - Perú para la realización del estudio en dicho lugar. Posteriormente, se comenzó a seleccionar a los pacientes según los criterios de inclusión y exclusión mencionados para luego realizarles las exodoncias, según técnica atraumática con periotomo (Sharma et al., 2015), y posteriormente distribuir los alvéolos post exodoncia de cada paciente en 2 grupos (Grupo A o control y Grupo B o experimental) de forma aleatoria por medio de un generador de númerosaleatorios por ordenador. A los alvéolos post exodoncia que pertenecen al Grupo A se les dejó con un coágulo de sangre para su curación normal y a los alvéolos post exodoncia del Grupo B se les administró FRP (como tapón y membrana), obtenido según el protocolo de Choukroun; para determinar el efecto del FRP en la curación de los tejidos blandos de los alvéolos post exodoncia atraumática se utilizó el índice de Landry (Landry et al., 1988).

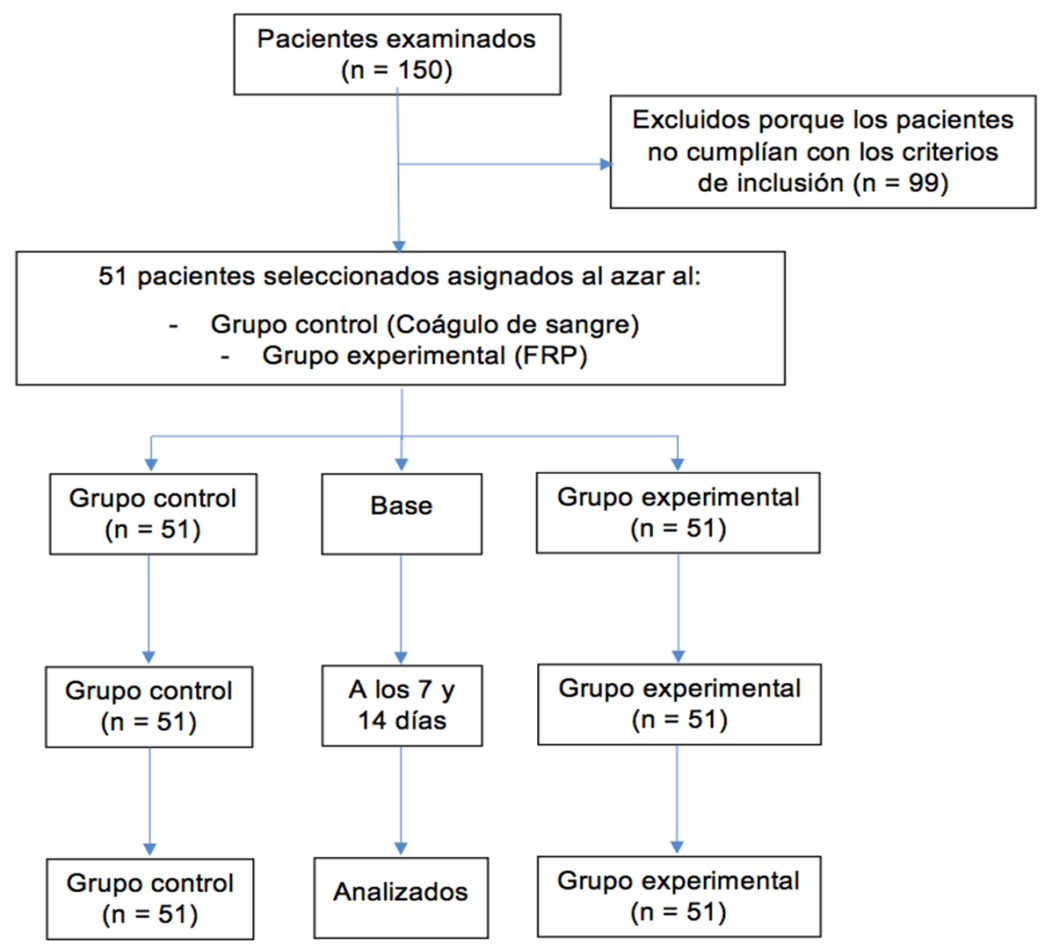

Fig. 1. Diagrama de flujo del estudio (según CONSORT).
Cada participante tuvo conocimiento de qué se trataba el estudio para lo cual cada uno firmó un consentimiento informado y leyó la hoja explicativa del estudio antes de empezar. Una vez realizado esto, el operador (MT), quien fue calibrado por un especialista en Periodoncia (AA) para la técnica de exodoncia atraumática como para la obtención y colocación de la FRP (Alfa de Cronbach $=0,8$ ), comenzó a realizar la exodoncia atraumática de cada pieza dental con materiales completamente estériles. Los participantes que alvéolo post exodoncia recibía o no el tratamiento con FRP.

Una vez terminada la cirugía se despidió al participante y se le citó a los 7 y 14días para sus controles. Posteriormente, en cada control, el examinador (MT) tuvo una ficha de recolección de datos que constaba de dos apartados: datos del participante (Sexo, edad y fecha) y un cuadro en donde se anotó los datos correspondientes al índice de Landry (Medidas a los 7 y 14días después de la cirugía), las cuales fueron expresadas en porcentajes.

Los datos recolectados fueron ingresados en una base de datos y posteriormente procesados empleando el programa SPSS 23.0. Para la comparación de cada una de las variables del estudio se utilizó el Test de Mc Nemar y el Test exacto de Fisher. La significación estadística fue del $5 \%$.

\section{RESULTADOS}

De los 150 pacientes examinados en un inicio, se eligieron 51 pacientes que cumplían con los criterios de inclusión y exclusión y fueron analizados hasta el final del estudio (Fig. 1).

La distribución de la muestra según su género y edad se puede observar en la Tabla I. Al comparar el efecto de la FRP en la curación de los tejidos blandos de alvéolos post exodoncia atraumática se encontró que existe una diferencia estadísticamente significativa a los 7 y 14días después de la cirugía $(p<0.05)$ (Tabla II). Al comparar el efecto de la FRP en la curación de los tejidos blandos de alvéolos post exodoncia atraumática según grupo 
TRAVEZÁN-MOREYRA, M.; AGUIRRE-AGUILAR, A. \& ARBILDO-VEGA, H. Efecto de la fibrina rica en plaquetas en la curación de los tejidos blandos de alveolos post exodoncia atraumática. Un ensayo clínico controlado aleatorizado cruzado a ciego simple. Int. J. Odontostomat., 15(1):240-247, 2021.

Tabla I. Distribución de pacientes según sexo y edad.

\begin{tabular}{clcr}
\hline & Variable & $\mathrm{n}$ & $\%$ \\
\hline \multirow{3}{*}{ Sexo } & Masculino & 16 & 31,37 \\
& Femenino & 35 & 68,63 \\
& $18-29$ años & 12 & 22,22 \\
\multirow{5}{*}{ Edad } & $30-59$ años & 12 & 22,22 \\
& 60 años a más & 27 & 55,56 \\
& TOTAL & 51 & 100 \\
\hline
\end{tabular}

etáreo se encontró que no existe una diferencia estadísticamente significativa a los 7 y 14 días después de la cirugía $(p>0,05)$ (Tabla III). Al comparar el efecto de la FRP en la curación de los tejidos blandos de alveolos post exodoncia atraumática según sexo se encontró que no existe una diferencia estadísticamente significativa a los 7 y 14días después de la cirugía $(p>0,05)$ (Tabla IV).

Tabla II. Efecto de la FRP en la curación de los tejidos blandos de alvéolos post exodoncia atraumática

\begin{tabular}{|c|c|c|c|c|c|c|}
\hline \multirow{3}{*}{ Periodo } & \multirow{3}{*}{ Cicatrización } & \multicolumn{4}{|c|}{ Grupos } & \multirow{3}{*}{$p^{*}$} \\
\hline & & \multicolumn{2}{|c|}{ Control } & \multicolumn{2}{|c|}{ Experimental } & \\
\hline & & $\mathrm{n}$ & $\%$ & $\mathrm{n}$ & $\%$ & \\
\hline \multirow{5}{*}{7 días } & Muy pobre & 5 & 9,8 & 0 & 0 & 0,02 \\
\hline & Pobre & 34 & 66,67 & 3 & 5,88 & 0,019 \\
\hline & Bueno & 12 & 23,53 & 30 & 58,82 & 0,019 \\
\hline & Muy bueno & 0 & 0 & 18 & 35,29 & 0,02 \\
\hline & Excelente & 0 & 0 & 0 & 0 & 0,02 \\
\hline \multirow{5}{*}{14 días } & Muy pobre & 0 & 0 & 0 & 0 & 0,003 \\
\hline & Pobre & 7 & 13,73 & 0 & 0 & 0,003 \\
\hline & Bueno & 35 & 68,63 & 1 & 1,96 & 0,003 \\
\hline & Muy bueno & 9 & 17,65 & 31 & 60,78 & 0,011 \\
\hline & Excelente & 0 & 0 & 19 & 37,25 & 0,003 \\
\hline
\end{tabular}

* Test de Mc Nemar.

Tabla III. Efecto de la FRP en la curación de los tejidos blandos de alvéolos post exodoncia atraumática según edad.

\begin{tabular}{|c|c|c|c|c|c|c|c|c|c|}
\hline \multirow[b]{2}{*}{ Periodo } & \multirow[b]{2}{*}{ Grupo } & \multirow[b]{2}{*}{$\begin{array}{l}\text { Grupo } \\
\text { etáreo }\end{array}$} & \multirow[b]{2}{*}{ Frecuencia } & \multirow[b]{2}{*}{$\begin{array}{l}\text { Muy } \\
\text { pobre }\end{array}$} & \multicolumn{3}{|c|}{ Cicatrización } & \multirow[b]{2}{*}{ Excelente } & \multirow[b]{2}{*}{$\mathrm{p}^{*}$} \\
\hline & & & & & Pobre & Bueno & $\begin{array}{c}\text { Muy } \\
\text { bueno }\end{array}$ & & \\
\hline \multirow{12}{*}{7 días } & \multirow{6}{*}{ Control } & $18-29$ & $\mathrm{n}$ & 3 & 6 & 3 & 0 & 0 & \multirow{6}{*}{0,335} \\
\hline & & $(n=12)$ & $\%$ & 25 & 50 & 25 & 0 & 0 & \\
\hline & & $30-59$ & $\mathrm{n}$ & 1 & 9 & 2 & 0 & 0 & \\
\hline & & $(n=12)$ & $\%$ & 8,33 & 75 & 16,67 & 0 & 0 & \\
\hline & & \multirow{2}{*}{$\begin{array}{c}60-+ \\
(n=27)\end{array}$} & $\mathrm{n}$ & 1 & 19 & 7 & 0 & 0 & \\
\hline & & & $\%$ & 3,7 & 70,37 & 25,93 & 0 & 0 & \\
\hline & \multirow{6}{*}{ Experimental } & $18-29$ & $\mathrm{n}$ & 0 & 0 & 4 & 8 & 0 & \multirow{6}{*}{0,076} \\
\hline & & $(n=12)$ & $\%$ & 0 & 0 & 33,33 & 66,67 & 0 & \\
\hline & & $30-59$ & $\mathrm{n}$ & 0 & 0 & 8 & 4 & 0 & \\
\hline & & $(n=12)$ & $\%$ & 0 & 0 & 66,67 & 33,33 & 0 & \\
\hline & & \multirow{2}{*}{$\begin{array}{c}60-+ \\
(n=27)\end{array}$} & $\mathrm{n}$ & 0 & 3 & 18 & 6 & 0 & \\
\hline & & & $\%$ & 0 & 11,11 & 66,67 & 22,22 & 0 & \\
\hline \multirow{12}{*}{14 días } & \multirow{6}{*}{ Control } & \multirow{2}{*}{$\begin{array}{l}18-29 \\
(n=12)\end{array}$} & $\mathrm{n}$ & 0 & 4 & 7 & 1 & 0 & \multirow{6}{*}{0,094} \\
\hline & & & $\%$ & 0 & 33,33 & 58,33 & 8,33 & 0 & \\
\hline & & $30-59$ & $\mathrm{n}$ & 0 & 2 & 9 & 1 & 0 & \\
\hline & & $(n=12)$ & $\%$ & 0 & 16,67 & 75 & 8,33 & 0 & \\
\hline & & \multirow{2}{*}{$\begin{array}{c}60-+ \\
(n=27)\end{array}$} & $\mathrm{n}$ & 0 & 1 & 19 & 7 & 0 & \\
\hline & & & $\%$ & 0 & 3,7 & 70,37 & 25,93 & 0 & \\
\hline & \multirow{6}{*}{ Experimental } & \multirow{2}{*}{$\begin{array}{l}18-29 \\
(n=12)\end{array}$} & $\mathrm{n}$ & 0 & 0 & 0 & 6 & 6 & \multirow{6}{*}{0,86} \\
\hline & & & $\%$ & 0 & 0 & 0 & 50 & 50 & \\
\hline & & $30-59$ & $\mathrm{n}$ & 0 & 0 & 0 & 8 & 4 & \\
\hline & & $(n=12)$ & $\%$ & 0 & 0 & 0 & 66,67 & 33,33 & \\
\hline & & \multirow{2}{*}{$\begin{array}{c}60-+ \\
(n=27)\end{array}$} & $\mathrm{n}$ & 0 & 0 & 1 & 17 & 9 & \\
\hline & & & $\%$ & 0 & 0 & 3,7 & 62,96 & 33,33 & \\
\hline
\end{tabular}

\footnotetext{
* Test exacto de Fisher.
} 
TRAVEZÁN-MOREYRA, M.; AGUIRRE-AGUILAR, A. \& ARBILDO-VEGA, H. Efecto de la fibrina rica en plaquetas en la curación de los tejidos blandos de alveolos post exodoncia atraumática. Un ensayo clínico controlado aleatorizado cruzado a ciego simple. Int. J. Odontostomat., 15(1):240-247, 2021.

Tabla IV. Efecto de la FRP en la curación de los tejidos blandos de alvéolos post exodoncia atraumática según sexo.

\begin{tabular}{|c|c|c|c|c|c|c|c|c|c|}
\hline \multirow[b]{2}{*}{ Periodo } & \multirow[b]{2}{*}{ Grupo } & \multirow[b]{2}{*}{$\begin{array}{l}\text { Grupo } \\
\text { etáreo }\end{array}$} & \multirow[b]{2}{*}{ Frecuencia } & \multicolumn{5}{|c|}{ Cicatrización } & \multirow[b]{2}{*}{$p^{*}$} \\
\hline & & & & $\begin{array}{l}\text { Muy } \\
\text { pobre }\end{array}$ & Pobre & Bueno & $\begin{array}{l}\text { Muy } \\
\text { bueno }\end{array}$ & Excelente & \\
\hline \multirow{8}{*}{7 días } & \multirow{4}{*}{ Control } & Masculino & $\mathrm{n}$ & 4 & 22 & 9 & 0 & 0 & \multirow{4}{*}{0,723} \\
\hline & & $(n=35)$ & $\%$ & 11,43 & 62,86 & 25,71 & 0 & 0 & \\
\hline & & \multirow{2}{*}{$\begin{array}{l}\text { Femenino } \\
(n=16)\end{array}$} & $\mathrm{n}$ & 1 & 12 & 3 & 0 & 0 & \\
\hline & & & $\%$ & 6,25 & 75 & 18,75 & 0 & 0 & \\
\hline & \multirow{4}{*}{ Experimental } & \multirow{2}{*}{$\begin{array}{l}\text { Masculino } \\
(n=35)\end{array}$} & $\mathrm{n}$ & 0 & 2 & 19 & 14 & 0 & \multirow{4}{*}{0,626} \\
\hline & & & $\%$ & 0 & 5,71 & 54,29 & 40 & 0 & \\
\hline & & \multirow{2}{*}{$\begin{array}{c}\text { Femenino } \\
(n=16)\end{array}$} & $\mathrm{n}$ & 0 & 1 & 11 & 4 & 0 & \\
\hline & & & $\%$ & 0 & 6,25 & 68,75 & 25 & 0 & \\
\hline \multirow{8}{*}{14 días } & \multirow{4}{*}{ Control } & \multirow{2}{*}{$\begin{array}{l}\text { Masculino } \\
(n=35)\end{array}$} & $\mathrm{n}$ & 0 & 5 & 24 & 6 & 0 & \multirow{4}{*}{1} \\
\hline & & & $\%$ & 0 & 14,29 & 68,57 & 17,14 & 0 & \\
\hline & & \multirow{2}{*}{$\begin{array}{l}\text { Femenino } \\
(\mathrm{n}=16)\end{array}$} & $\mathrm{n}$ & 0 & 2 & 11 & 3 & 0 & \\
\hline & & & $\%$ & 0 & 12,5 & 68,75 & 18,75 & 0 & \\
\hline & \multirow{4}{*}{ Experimental } & \multirow{2}{*}{$\begin{array}{l}\text { Masculino } \\
(\mathrm{n}=35)\end{array}$} & $\mathrm{n}$ & 0 & 0 & 1 & 20 & 14 & \multirow{4}{*}{0,691} \\
\hline & & & $\%$ & 0 & 0 & 2,86 & 57,14 & 40 & \\
\hline & & \multirow{2}{*}{$\begin{array}{l}\text { Femenino } \\
(n=16)\end{array}$} & $\mathrm{n}$ & 0 & 0 & 0 & 11 & 5 & \\
\hline & & & $\%$ & 0 & 0 & 0 & 68,75 & 31,25 & \\
\hline
\end{tabular}

* Test exacto de Fisher.

\section{DISCUSIÓN}

En el presente ensayo clínico aleatorizado se encontró una mayor curación de los tejidos blandos en los alvéolos post exodoncia atraumática al colocar la FRP la cual fue estadísticamente significativa.

Se han realizado numerosos estudios e investigaciones sobre los procesos de curación de los alvéolos post exodoncia; sin embargo, la mayoría de estos estudios son histológicos. La FRP es un concentrado de plaquetas y es una matriz similar a un gel que contiene una alta concentración de plaquetas intactas, no activadas e inactivadas, contenidas dentro de una matriz de fibrina que liberan una concentración relativamente constante de factores de crecimiento, durante un período de 7 días, para la curación de tejidos duros y blandos. La razón científica detrás del uso de ésta preparación radica en el hecho de que las plaquetas sirven como un depósito de muchos factores de crecimiento, descritos por primera vez por Ross et al. (1974) que se sabe que desempeñan un papel crucial en el proceso de curación de tejidos duros y blandos. La FRP estimula la proliferación osteoblástica humana, y la histología ha demostrado que tiene un efecto sobre la neoangiogénesis. En forma de membrana, se puede utilizar como una malla de fibrina, que sirve como matriz para acelerar la cicatrización de los márgenes de la herida y es relativamente económico, de fácil, rápido y simple procesamiento. Al rellenar unalvéolo post exodonciacon la FRP, la neovascularización se establece a través del coágulo de la FRP y se desarrolla una cubierta epitelial. A pesar del potencial infeccioso e inflamatorio de los alvéolos post exodoncia, la curación rápida de la herida al usar FRP ocurre sin dolor, inflamación y otros signos de inflamación y procesos infecciosos (Ross et al.; Mosesson, 2005; Boyapati \& Wang, 2006; Choukroun et al., 2006; Dohan Ehrenfest et al., 2009; Mazor \& Horowitz, 2009; He \& Lin, 2009; Blair \& Flaumenhaft, 2009; Dohan Ehrenfest et al., 2010; Gurbuzer \& Pikdoken, 2010; Gassling et al., 2010; Chang \& Zhao, 2011; Jiing et al.; Kang et al., 2011; Bielecki \& Dohan Ehrenfest, 2012; Del Corso et al., 2012a; Dohan Ehrenfest et al., 2012; Bielecki et al., 2012; Simonpieri et al., 2012; Zumstein et al., 2012; Marenzi et al., 2015; Dutta et al., 2016; Alzahrani et al.; Srinivas et al.). Los resultados encontrados en el presente estudio fueron similares a los encontrados por otros estudios (Moya-Villaescusa \& Sánchez-Pérez, 2010; Marenzi et al.; Dutta et al.; Alzahrani et al.; Srinivas et al.), quienes mostraron que la FRP estimulaba el proceso de curación de tejidos blandos cuando se aplicaba a los alvéolos post exodoncia recientes.

Un alvéolo post exodoncia contiene tanto tejidos duros como blandos que se curan concomitantemente con un cierto retraso en los procesos de curación. Hacia el final de la primera semana, el coá- 
TRAVEZÁN-MOREYRA, M.; AGUIRRE-AGUILAR, A. \& ARBILDO-VEGA, H. Efecto de la fibrina rica en plaquetas en la curación de los tejidos blandos de alveolos post exodoncia atraumática. Un ensayo clínico controlado aleatorizado cruzado a ciego simple. Int. J. Odontostomat., 15(1):240-247, 2021.

gulo se retrae y se descompone siendo reemplazado por un tejido de granulación. El tejido de granulación cubre la parte superior de la cresta del hueso alveolar, y el epitelio prolifera a lo largo de los márgenes de la herida del tejido blando. La FRP en comparación con otros concentrados de plaquetas, no requiere agentes anticoagulantes o gelificantes, y el coágulo de la FRP se forma naturalmente teniendo una arquitectura tridimensional densa y compleja que contiene no solo plaquetas sino también leucocitos (Del Corso et al., 2012b; Srinivas et al.).

Las puntuaciones del índice de curación se basan en el eritema existente, la presencia de tejido de granulación, el sangrado, la supuración y la epitelización. En el presente estudio, durante las visitas de seguimiento postoperatorias, las heridas quirúrgicas creadas se evaluaron mediante el índice de Landry y se obtuvo una puntuación que osciló entre muy pobre y excelente. La evaluación promedio del cambio de tejidos blandos mostró que una proporción significativamente mayor de sitios del grupo con FRP a los 14 días postoperatorio, mostraron un mejor índice de curación en comparación con los sitios del grupo control.

Las características de la muestra estudiada, en cuanto al sexo, presentan una heterogeneidad; ya que existe un mayor número de mujeres que de hombres. El predominio de este grupo es debido posiblemente a que en el periodo de realización del presente estudio, acudieron al Servicio de Odontología del Hospital Distrital Santa Isabel del Porvenir - Perú más mujeres que hombres o quienes más cumplían los criterios de selección eran las mujeres.

En relación con la edad, la muestra, presenta también una heterogeneidad; ya que existe un mayor predominio de pacientes mayores de 60 años. El predominio de este grupo es debido posiblemente a que las personas en ese grupo etáreo presentaron mayores molestias en piezas dentales que se encontraban para realizar exodoncia.

En cuanto al género y la edad, se observa que tanto en hombres como en mujeres y en jóvenes como en adultos y en adultos mayores existe una mayor curación de los tejidos blandos en los alvéolos post exodoncia atraumática al colocar la FRP. Esto nos da a entender que la FRP presente un efecto positivo de forma independiente del sexo y la edad, y esto posiblemente sea por el efecto añadido que proporciona la FRP.
Una limitación del presente estudio fue el corto tiempo de seguimiento para la curación de un alvéolo post exodoncia y el tipo de exodoncia que fue simple. A pesar de ello, se pudo observar la ventaja que proporciona el FRP a los tejidos blando de alvéolos post exodoncia y sobre todo de exodoncias atraumáticas. Adicionalmente, los autores creemos que este estudio es un inicio para la realización de futuras investigaciones que presenten un tiempo de seguimiento mayor o igual a 3 meses y la evaluación de los tejidos duros en ese mismo periodo de tiempo.

\section{CONCLUSIÓN}

La FRP presenta un efecto positivo en la curación de los tejidos blandos de alvéolos post exodoncia atraumática de forma independiente del sexo y la edad.

TRAVEZÁN-MOREYRA, M.; AGUIRRE-AGUILAR, A. \& ARBILDO-VEGA, H. Effect of the platelet-rich fibrin on the healing of the soft tissues of sockets after atraumatic exodontics. A single-blind cross-randomized controlled clinical trial. Int. J. Odontostomat., 15(1):240-247, 2021.

ABSTRACT: The objective of the study was to determine the effect of platelet-rich fibrin (PRF) on the healing of soft tissues of socket after atraumatic exodontia. The present is a single-blind, cross-sectional randomized controlled trial. It was carried out in the Dental Service of the Santa Isabel District Hospital of Porvenir - Peru, during the years 2016 and 2017. The sample consisted of 51 patients whose alveoli post exodontia were randomly divided into 2 groups, each patient signed an informed consent. Group A (control) was left with a blood clot for normal healing and group B (experimental) was given PRF (as a plug and membrane), obtained according to the Choukroun protocol. The effect of PRF on the healing of the soft tissues of the alveoli after atraumatic exodontia was used the Landry index. The measurements were made at 7 and 14 days after surgery. For the comparison of each one of the variables of the study, the Mc Nemar test and the Fisher exact test were used. The statistical significance was $5 \%$. When comparing both groups in the healing of the soft tissues of alveoli after atraumatic exodontia, a statistically significant difference was found at 7 and 14 days after surgery $(p<0.05)$. When comparing both groups in the healing of soft tissues of alveoli after atraumatic exodontia according to age group and sex, it was found that there is no statistically significant difference at 7 and 14 days after surgery $(p>0.05)$. The PRF has a positive effect on the healing of the soft tissues of the alveoli after atraumatic exodontia independently of sex and age.

KEY WORDS: platelet-rich fibrin, PRF, socket extraction, wound healing, soft tissues. 


\section{REFERENCIAS BIBLIOGRAFICAS}

Alzahrani, A. A.; Murriky, A. \& Shafik, S. Influence of platelet rich fibrin on post-extraction socket healing: A clinical and radiographic study. Saudi Dent. J., 29(4):149-55, 2017.

Araujo, M. G. \& Lindhe, J. Dimensional ridge alterations following tooth extraction: An experimental study in dog. J. Clin. Periodontol., 32(2):212-8, 2005.

Bielecki, T. \& Dohan Ehrenfest, D. M. Leukocyte-and platelet-rich plasma (L-PRP)/fibrin (L-PRF) in medicine - past, present, future. Curr. Pharm. Biotechnol., 13(7):i-ii, 2012.

Bielecki, T.; Dohan Ehrenfest, D. M.; Everts, P. A. \& Wiczkowski, A. The role of leukocytes from L-PRP/L-PRF in wound healing an immune defense: new perspectives. Curr. Pharm. Biotechnol., 13(7):1153-62, 2012.

Blair, P. \& Flaumenhaft, R. Platelet a/pha-granules: basic biology and clinical correlates. Blood Rev., 23(4):177-89, 2009.

Boyapati, L. \& Wang, H. L. The role of platelet-rich plasma in sinus sugmentation: a critical review. Implant. Dent., 15(2):160-70, 2006.

Buchwald, S. \& Kocher, T. Tooth loss and periodontitis by socioeconomic status and inflammation in a longitudinal populationbased study. J. Clin. Periodontol., 40(3):203-11, 2013.

Cardaropoli, G. \& Araujo, M. Dynamics of bone tissue formation in tooth extraction sites: An experimental study in dogs. J. Clin. Periodontol., 30(9):809-18, 2003.

Chan, H. L.; Lin, G. H.; Fu, J. H. \& Wang, H. L. Alterations in bone quality after socket preservation with grafting materials: A systematic review. Int. J. Oral Maxillofac. Implants, 28(3):71020, 2013

Chang, Y. C. \& Zhao, J. H. Effects of platelet-rich fibrin on human periodontal ligament fibroblasts and application for periodontal infrabony defects. Aust. Dent. J., 56(4):365-71, 2011.

Choukroun, J. \& Diss, A. Platelet-rich fibrin (PRF): a secondgeneration platelet concentration. Part V: histologic evaluations of PRF effects on bone allograft maturation in sinus lift. Oral Surg. Oral Med. Oral Pathol. Oral Radiol. Endod., 101(3):299303, 2006.

Choukroun, J.; Diss, A.; Simonpieri, A.; Girard, M. O.; Schoeffler, C.; Dohan, S. L.; Dohan, A. J.; Mouhyi, J. \& Dohan, D. M. Plateletrich fibrin (PRF): a second-generation platelet concentrate. Part IV: clinical effects on tissue healing. Oral Surg. Oral Med. Oral Pathol. Oral Radiol. Endod., 101(3):e56-60, 2006.

Del Corso, M.; Mazor, Z.; Rutkowski, J. L. \& Dohan Ehrenfest, D. M. The use of leucocyte-and platelet-rich fibrin during immediate postextractive implantation and loading for the esthetic replacement of a fractured maxillary central incisor. J. Oral Implantol., 38(2):181-7, 2012.

Del Corso, M.; Vervelle, A.; Simonpieri, A.; Jimbo, R.; Inchingolo, F.; Sammartino, G. \& Dohan Ehrenfest, D. M. Current knowledge and perspectives for the use of platelet-rich plasma (PRP) and platelet-rich fibrin (PRF) In oral and maxillofacial surgery. Part 1: Periodontal and dentoalveolar surgery. Curr. Pharm. Biotechnol., 13(7):1207-30, 2012.

Dohan Ehrenfest, D. M.; Bielecki, T.; Jimbo, R.; Barbé, G.; Del Corso, M.; Inchingolo, F. \& Sammartino, G. Do the fibrin architecture and leukocyte content influence the growth factor release of platelet concentrates? An evidence-based answer comparing a pure platelet-rich plasma (P-PRP) gel and leukocyte-and plateletrich fibrin (L-PRF). Curr. Pharm. Biotechnol., 13(7):1145-52, 2012.

Dohan Ehrenfest, D. M.; de Peppo, G. M.; Doglioli, P. \& Sammartino, G. Slow release of growth factors and thrombospodin-1 in Choukroun's platelet-rich fibrin (PRF): a gold standard to achieve for all surgical platelet concentrates technologies. Growth Factors, 27(1):63-9, 2009.
Dohan Ehrenfest, D. M.; Del Corso, M.; Inchingolo, F. \& Charrier, J. B. Selecting a relevant in vitro cell model for testing and comparing the effects of a Choukroun's platelet-rich fibrin (PRF) membrane and platelet-rich plasma (PRP) gel: tricks and traps. Oral Surg. Oral Med. Oral Pathol. Oral Radiol. Endod., 110(4):409-11, 2010.

Dohan, D. M. \& Choukroun, J. Platelet-rich fibrin (PRF): a secondgeneration platelet concentration. Part II: platelet-related biologic features. Oral Surg. Oral Med. Oral Pathol. Oral Radiol. Endod., 101(3):e45-50, 2006.

Dutta, S. R.; Passi, D.; Singh, P.; Sharma, S.; Singh, M. \& Srivastavas, D. A randomized comparative prospective study of platelet-rich plasma, platelet-rich fibrin, and hydroxyapatite as a graft material for mandibular third molar extraction socket healing. Natl. J. Maxillofac. Surg., 7(1):45-51, 2016.

Forum, S. \& Cho, S. C. Histological comparison of healing extraction sockets implanted with bioactive glass or demineralized freezedried bone allograft: a pilot study. J. Periodontol., 73(1):94-102, 2002.

Gassling, V.; Douglas, T.; Warnke, P. H.; Açil, Y.; Wiltfang, J. \& Becker, S. T. Platelet-rich fibrin membranes as scaffolds for periosteal tissue engineering. Clin. Oral Implants Res., 21(5):543-9, 2010.

Gonda, T. \& Mac Entee, M. I. Predictors of multiple tooth loss among socioculturally diverse elderly subjects. Int. J. Prosthodont., 26(2):127-34, 2013

Gurbuzer, B. \& Pikdoken, L. Scintigraphic evaluation of osteoblastic activity in extraction sockets treated with platelet-rich fibrin. $J$. Oral Maxillofac. Surg., 68(5):980-9, 2010.

He, L. \& Lin, Y. A comparative study of platelet-rich fibrin (PRF) and platelet-rich plasma (PRP) on the effect of proliferation and differentiation of rat osteoblasts in vitro. Oral Surg. Oral Med. Oral Phatol. Oral Radiol. Endod., 108(5):707-13, 2009.

Jiing, H. Z.; Chung, H. T. \& Yu, C. C. Clinical and histologic evaluation of healing in an extraction socket filled with platelet-rich fibrin. $J$. Dent. Sci., 6(2):116-22, 2011.

Kang, Y. H.; Jeon, S. H.; Park, J. Y.; Chung, J. H.; Choung, Y. H.; Choung, H. W.; Kim, E. S. \& Choung, P. H. Platelet-rich fibrin is a bioscaffold and reservoir of growth factors for tissue regeneration. Tissue Eng. Part. A., 17(3-4):349-59, 2011.

Kutkut, A. \& Andreana, S. Extraction socket preservation graft before implant placement with calcium sulfate hemihydrate and plateletrich plasma: a clinical and histomorphometric study in humans. J. Periodontol., 83(4):401-9, 2012.

Landry, R. G.; Turnbull, R. S. \& Howley, T. Effectiveness of benzydamyne $\mathrm{HCl}$ in the treatment of periodontal post surgical patients. Res. Clin. Forums, 10:105-18, 1988.

Marelli, M. \& Tatullo, M. Influence of PRF in the healing of bone and gingival tissues. Clinical and histological evaluations. Eur. Rev. Med. Pharmacol. Sci., 17(14):1958-62, 2013.

Marenzi, G.; Riccitiello, F.; Tia, M.; di Lauro, A. \& Sammartino, G. influence of leukocyte-and platelet-rich fibrin (L-PRF) in the healing of simple postextraction sockets: A split-mouth study. Biomed. Res. Int., 2015:369273, 2015.

Mazor, Z. \& Horowitz, R. A. Sinus floor augmentation with simultaneous implant placement using Choukroun's plateletrich fibrin as the sole grafting material: a radiologic and histologic study at 6 months. J. Periodontol., 80(12):2056-64, 2009.

Mosesson, M. W. Fibrinogen and fibrin structure and functions. J. Thromb. Haemost., 3(8):1894-904, 2005.

Moya-Villaescusa, M. J. \& Sánchez-Pérez, A. Measurement of ridge alterations following tooth removal: a radiographic study in humans. Clin. Oral Implants Res., 21(2):237-42, 2010.

Norton, M. R. \& Wilson, J. Dental implants placed in extraction sites implanted with bioactive glass: human histologic and clinical outcome. Int. J. Oral Maxillofac. Implants, 17(2):249$57,2002$. 
TRAVEZÁN-MOREYRA, M.; AGUIRRE-AGUILAR, A. \& ARBILDO-VEGA, H. Efecto de la fibrina rica en plaquetas en la curación de los tejidos blandos de alveolos post exodoncia atraumática. Un ensayo clínico controlado aleatorizado cruzado a ciego simple. Int. J. Odontostomat., 15(1):240-247, 2021.

Ross, R.; Glomset, J.; Kariya, B. \& Harker, L. A platelet-dependent serum factor that stimulates the proliferation of arterial smooth muscle cells in vitro. Proc. Natl. Acad. Sci. U. S. A., 71(4):120710, 1974.

Rutherford, R. B. \& Niekrash, C. E. Platelet-derived and insulin-like growth factors stimulate regeneration of periodontal attachment in monkeys. J. Periodontal. Res., 27(4 Pt. 1):285-90, 1992.

Sharma, S. D.; Vidya, B.; Alexander, M. \& Deshmukh, S. Periotome as an aid to atraumatic extraction: a comparative double blind randomized controlled trial. J. Maxillofac. Oral Surg., 14(3):6115, 2015.

Simonpieri, A.; Del Corso, M.; Sammartino, G. \& Dohan Ehrenfest, D. M. The relevance of Choukrouns's platelet-rich fibrin and metronidazole during complex maxillary rehabilitations using bone allograft. Part II: implant surgery, prosthodontics, and survival. Implant Dent., 18(3):220-9, 2009.

Simonpieri, A.; Del Corso, M.; Vervelle, A.; Jimbo, R.; Inchingolo, F.; Sammartino, G. \& Dohan Ehrenfest, D. M. Current knowledge and perspectives for the use of platelet-rich plasma (PRP) and platelet-rich fibrin (PRF) in oral and maxillofacial surgery. Part 2: bone graft, implant and reconstructive surgery. Curr. Pharm. Biotechnol., 13(7):1231-56, 2012.

Srinivas, B.; Das, P.; Rana, M. M.; Qureshi, A. Q.; Vaidya, K. C. \& Ahmed Raziuddin, S. J. Wound healing and bone regeneration in postextraction sockets with and without platelet-rich fibrin. Ann. Maxillofac. Surg., 8(1):28-34, 2018.

Vance, G. S. \& Greenwell, H. Comparison of an allograft in an experimental putty carrier and a bovine-derived xenograft used in ridge preservation: a clinical and histologic study in humans. Int. J. Oral Maxillofac. Implants, 19(4):491-7, 2004.

Zhang, Y. \& Wang, Y. A platelet-derived growth factor releasing chitosan/coral composite scaffold for periodontal tissue engineering. Biomaterials, 28(8):1515-22, 2007.

Zumstein, M. A.; Berger, S.; Schober, M.; Boileau, P.; Nyffeler, R. W.; Horn, M. \& Dahinden, C. A. Leukocyte-and platelet-rich fibrin (L-PRF) for long-term delivery of growth factor in rotator cuff repair: review, preliminary results and future directions. Curr. Pharm. Biotechnol., 13(7):1196-206, 2012.
Dirección de correspondencia:

Heber Isac Arbildo Vega

Facultad de Odontología

Universidad San Martín de Porres

Av. Húsares de Junín 611

Trujillo

PERÚ

E-mail: hiav666@hotmail.com hiav30@gmail.com 